\title{
Screening of different Rice entries against Rice Gall Midge, Orseolia oryzae (Wood-Mason)
}

\author{
Atanu Seni*, Bhima Sen Naik
}

Orissa University of Agriculture and Technology, AICRIP, RRTTS, Chiplima-768025, Sambalpur, Odisha

\begin{abstract}
In order to develop rice cultivars for resistance to the gall midge, Orseolia oryzae (Wood-Mason), some rice entries were screened under natural field conditions at the Chiplima, OUAT, Odisha under All India Coordinated Rice Improvement Project during kharif 2016. Gall midge incidence as silver shoot was recorded on 30 and 50 days after transplanting and scoring was done. Highest incidence of silver shoot was recorded in TN-1 (36.71\% SS after 50 DAT) whereas 12 entries viz., W 1263, INRC 3021, Sudu Hondarawala, PTB 26, RP 4686-48-1-937, RMSG-11, WGL 1147, WGL 1127, WGL 1121, WGL 1131, WGL 1141, JGL 27058 were found resistant to gall midge damage. Based on the reaction of the different entries the presence of biotype 1 was identified.
\end{abstract}

Keywords-Gall midge, Chiplima, rice entries, screening, field.

\section{INTRODUCTION}

Rice is the most important cereal food crop of India covering about one-fourth of the total cropped area and providing food to about half of the Indian population. Introduction and wide adoption of high yielding varieties has led to severe incidence of different insect pests. Nearly 300 species of insect pests attack the rice crop at different stages and among them only 23 species cause notable damage [6]. Among them, Asian rice gall midge (GM), Orseolia oryzae (Wood-Mason) is one of the important insect which has been prevalent in almost all the rice growing states in India except the Western Uttar Pradesh, Uttaranchal, Punjab, Haryana and Hill states of Himachal Pradesh and Jammu and Kashmir [1]. GM causes an annual yield loss of $0.8 \%$ of the total production, amounting to US\$80 million [5]. This is essentially a monsoon pest and causes damage wherever high humidity and moderate temperature prevail, even in dry seasons [3]. The external symptom of damage caused by gall midge is the production of a silvery-white, tubular leaf sheath gall called a silver shoot or onion shoot. This is due to the feeding and salivary secretion by the larvae which turn the growing shoot meristem into a gall [7]. This renders the tiller sterile and do not bear panicle [9]. Many management strategies viz., chemical, cultural, biological and planting of resistant cultivars that have resistance to insects are employed to reduce the damage caused by this insect-pest. Among them, the use of resistant rice varieties appears to offer the most effective component for incorporation into an integrated pest management strategy [11]. For this, breeding resistant varieties has been a viable, ecologically acceptable approach for managing this pest [5].

\section{MATERIALS AND METHODS}

The experiment was conducted in the experimental farm of Regional Research and Technology Transfer Station (OUAT), Chiplima, Sambalpur, Odisha, during kharif, 2016. The Station is situated at $20^{0} 21^{\prime}$ N latitude and $80^{0} 55^{\prime} \mathrm{E}$ longitude in Dhankauda block of Sambalpur district at an altitude of $178.8 \mathrm{~m}$ above MSL. The climate of the area is warm/sub humid. Nursery of different rice entries were sown in the July and transplanting was done after 25 days of sowing at $15 \mathrm{~cm}$ x $15 \mathrm{~cm}$ hill spacing. All the agronomic practices were followed during crop growth period. Gall midge incidence as silver shoot was recorded on 30 and 50 days after transplanting and then percentage of silver shoot was worked out. The pest intensity was scored as per standard evaluation system, IRRI for gall midge.

Table.1: Standard evaluation system for rice gall midge

\begin{tabular}{|c|l|l|}
\hline Scale & Damage (\%) & Reaction \\
\hline 0 & No damage & HR \\
\hline 1 & $<1 \%$ & R \\
\hline 3 & $1-5 \%$ & MR \\
\hline 5 & $6-10 \%$ & $\mathrm{MS}$ \\
\hline 7 & $11-25 \%$ & $\mathrm{~S}$ \\
\hline 9 & $>25 \%$ & $\mathrm{HS}$ \\
\hline
\end{tabular}

\section{RESULTS AND DISCUSSION}

Rice gall midge is one of the major pestsof rice in Hirakud command area, Sambalpur, Odisha. Different rice entries obtained from ICAR-IIRR, Hyderabad were evaluated to 
find out the field resistance against rice gall midge. Rice gall midge is one of the major and regular pests of rice in Hirakud command area, Sambalpur, Odisha. The Sambalpur district in the west-central table land zone of Odisha is also considered to be the endemic pocket for gall midge in the state [2]. Different rice entries obtained from ICAR-IIRR, Hyderabad were evaluated to find out the field resistance against rice gall midge.

Among 164 entries screened against gall midge, the entries viz., W 1263, INRC 3021, Sudu Hondarawala, PTB 26, RP 4686-48-1-937, RMSG-11, WGL 1147, WGL 1127, WGL 1121, WGL 1131, WGL 1141, JGL 27058 were found resistant to gall midge damage. The entries viz., KAVYA, Aganni, INRC 15888, KAKAI (K 1417), SINNA SIVAPPU, PTB 12, PTB 32, TH BR 68, TH BR 69, TH BR 70, TH BR 71, TH BR 72, TH BR 74, TH BR 79, RMSG-7, RMSG-10, RP 5925-24, WGL 1143, WGL 1144, WGL 1145, WGL 1146, WGL 1118, WGL 1119, RP 2068-18-3-
5, JGL 21789, JGL 21831, JGL 25154, JGL 25969, JGL 27015, JGL 27020, JGL 27056, JGL 27075, JGL 27361, JGL 27371, KNM 1592, KNM 1598, KNM 1621, KNM 2251, KNM 2275, KNM 2266, KNM 13595, KNM 1623, KNM 1638, JGL 3828, and WGL 505 were found to be moderately resistant to gall midge incidence. The entries viz., Phalguna, Dukong 1, RP 2333-156-8, Abhaya, CAUR1, RP5923, COGR-2, IC 462362, IC 577588, ACC 4740, ACC 5403, IC 576897, IC 577224, IIRR-Bio-SB-6, ARC 15570C, VELLAI ILANKALAYAN, WGL32100, JGL 24520, JGL 25947, JGL 25960, JGL 25964, JGL 26989, JGL 27072, KNM 1717, KNM 1728, WGL 667, WGL 705, WGL 767 and TN 1 were found highly susceptible and the remaining entries were found susceptible to gall midge damage. Based on the reaction pattern of resistantsusceptible-susceptible (R-S-S) the prevalence of biotype 1 was identified at Sambalpur.

Table.2: Reaction of different rice entries against rice gall midge

\begin{tabular}{|l|l|c|c|l|}
\hline \multirow{2}{*}{ Entry No. } & \multirow{2}{*}{ Name of entry } & \multicolumn{2}{|c|}{ Silver shoot (SS) } & \multirow{2}{*}{ Reaction } \\
\cline { 3 - 4 } & & 30 DAT & 50 DAT & \\
\hline 1 & KAVYA & 1.37 & 1.96 & MR \\
\hline 3 & W 1263 & 0.57 & 0.00 & R \\
\hline 4 & ARC 6605 & 3.92 & 7.04 & MS \\
\hline 5 & PHALGUNA & 15.23 & 32.03 & HS \\
\hline 6 & ARC 5984 & 6.11 & 18.08 & S \\
\hline 7 & DUKONG 1 & 13.10 & 35.48 & HS \\
\hline 8 & RP 2333-156-8 & 15.00 & 25.87 & HS \\
\hline 9 & MADHURI L 9 & 2.50 & 5.71 & MS \\
\hline 10 & BG 380-2 & 16.55 & 27.70 & HS \\
\hline 11 & MR 1523 & 12.12 & 25.00 & S \\
\hline 12 & RP 2068-18-3-5 & 6.54 & 13.79 & S \\
\hline 13 & ABHAYA & 16.50 & 31.02 & HS \\
\hline 14 & INRC 3021 & 0.50 & 0.00 & R \\
\hline 15 & AGANNI & 0.00 & 1.21 & MR \\
\hline 16 & INRC 15888 & 1.84 & 2.36 & MR \\
\hline 17 & B 95-1 & 6.63 & 2.90 & MS \\
\hline 18 & TN1 & 17.62 & 36.71 & HS \\
\hline 19 & CAUR-1 & 31.16 & 39.74 & HS \\
\hline 20 & RP5923 & 30.84 & 43.48 & HS \\
\hline 21 & COGR-2 & 25.17 & 31.87 & HS \\
\hline 22 & IC 462248 & 17.12 & 16.00 & S \\
\hline 23 & IC 462362 & 46.15 & 46.36 & HS \\
\hline 24 & IC 463334 & 12.41 & 1.34 & S \\
\hline 25 & IC 463414 & 17.33 & 19.05 & S \\
\hline 26 & IC 463393 & IC 462447 & 19.14 & S \\
\hline
\end{tabular}




\begin{tabular}{|c|c|c|c|c|}
\hline 27 & IC 463987 & 21.99 & 14.67 & $\mathrm{~S}$ \\
\hline 28 & IC 577588 & 28.66 & 50.00 & HS \\
\hline 29 & ACC 3643 & 18.92 & 13.64 & $\mathrm{~S}$ \\
\hline 30 & ACC 4656 & 13.48 & 15.98 & $\mathrm{~S}$ \\
\hline 31 & ACC 4740 & 41.62 & 34.54 & HS \\
\hline 32 & ACC 5403 & 33.77 & 37.35 & HS \\
\hline 33 & IC 545528 & 16.18 & 20.74 & $\mathrm{~S}$ \\
\hline 34 & IC 576897 & 26.72 & 38.58 & HS \\
\hline 35 & IC 462336 & 21.38 & 16.40 & $\mathrm{~S}$ \\
\hline 36 & IC 545441 & 20.42 & 16.22 & $\mathrm{~S}$ \\
\hline 37 & IC 459646 & 14.39 & 11.18 & $\mathrm{~S}$ \\
\hline 38 & IC 450029 & 20.23 & 10.61 & $\mathrm{~S}$ \\
\hline 39 & IC 462336 & 19.12 & 19.40 & $\mathrm{~S}$ \\
\hline 40 & IC 577224 & 48.78 & 43.27 & HS \\
\hline 41 & IC 466408 & 14.38 & 6.99 & MS \\
\hline 42 & IIRR-Bio-SB-6 & 47.67 & 30.84 & HS \\
\hline 43 & AC 4236 & 17.16 & 7.14 & $\mathrm{~S}$ \\
\hline 44 & ARC 10676 & 21.53 & 3.87 & $\mathrm{~S}$ \\
\hline 45 & ARC 10840 & 17.19 & 20.16 & $\mathrm{~S}$ \\
\hline 46 & ARC 11220 & 12.82 & 15.85 & $\mathrm{~S}$ \\
\hline 47 & ARC 11281 & 20.11 & 17.46 & $\mathrm{~S}$ \\
\hline 48 & ARC 14636 & 19.28 & 19.77 & $\mathrm{~S}$ \\
\hline 49 & ARC 14771 & 25.73 & 17.39 & $\mathrm{~S}$ \\
\hline 50 & ARC $15570 \mathrm{C}$ & 25.74 & 37.91 & HS \\
\hline 51 & ARC 5754 & 14.93 & 18.71 & $\mathrm{~S}$ \\
\hline 52 & ARC 5956 & 14.53 & 7.03 & $\mathrm{~S}$ \\
\hline 53 & ARC 5981 & 9.83 & 17.65 & $\mathrm{~S}$ \\
\hline 54 & ASD 7 & 9.20 & 3.88 & MS \\
\hline 55 & KAKAI (K 1417) & 2.21 & 0.71 & MR \\
\hline 56 & SINNA SIVAPPU & 2.10 & 0.00 & MR \\
\hline 57 & SUDU HONDARAWALA & 1.09 & 0.00 & $\mathrm{R}$ \\
\hline 58 & PTB 12 & 3.10 & 0.80 & MR \\
\hline 59 & VELLAI ILANKALAYAN & 26.42 & 25.42 & HS \\
\hline 60 & ARC 6248 & 11.30 & 20.62 & $\mathrm{~S}$ \\
\hline 61 & CVL (CHINA) & 4.32 & 8.39 & MS \\
\hline 62 & ARC 6031-B & 6.90 & 1.57 & MS \\
\hline 63 & PTB 26 & 0.66 & 0.74 & $\mathrm{R}$ \\
\hline 64 & PTB 32 & 3.73 & 0.00 & MR \\
\hline 65 & IC 332045 & 11.73 & 1.55 & $\mathrm{~S}$ \\
\hline 66 & IC 466451 & 7.87 & 0.00 & MS \\
\hline 67 & TH BR 68 & 2.48 & 0.68 & MR \\
\hline 68 & TH BR 69 & 0.72 & 1.77 & MR \\
\hline 69 & TH BR 70 & 4.11 & 0.00 & MR \\
\hline 70 & TH BR 71 & 3.55 & 0.00 & MR \\
\hline 71 & TH BR 72 & 3.83 & 0.00 & MR \\
\hline 72 & TH BR 74 & 5.48 & 0.82 & MR \\
\hline 73 & TH BR 79 & 5.77 & 3.85 & MR \\
\hline
\end{tabular}




\begin{tabular}{|c|c|c|c|c|}
\hline 74 & RP4686-48-1-937 & 0.60 & 0.00 & $\mathrm{R}$ \\
\hline 75 & RMSG-2 & 13.69 & 15.75 & $\mathrm{~S}$ \\
\hline 76 & RMSG-5 & 20.61 & 22.47 & $\mathrm{~S}$ \\
\hline 77 & RMSG-6 & 6.90 & 7.58 & MS \\
\hline 78 & RMSG-7 & 2.03 & 0.60 & MR \\
\hline 79 & RMSG-10 & 1.37 & 0.00 & MR \\
\hline 80 & RMSG-11 & 1.08 & 0.00 & $\mathrm{R}$ \\
\hline 81 & RP 5925-24 & 1.55 & 0.00 & MR \\
\hline 82 & WGL 1143 & 1.44 & 0.83 & MR \\
\hline 83 & WGL 1144 & 1.80 & 0.00 & MR \\
\hline 84 & WGL 1145 & 1.28 & 0.00 & MR \\
\hline 85 & WGL 1146 & 1.12 & 0.00 & MR \\
\hline 86 & WGL 1147 & 0.00 & 0.72 & $\mathrm{R}$ \\
\hline 87 & WGL1118 & 1.29 & 0.00 & MR \\
\hline 88 & WGL1119 & 1.69 & 0.00 & MR \\
\hline 89 & WGL1121 & 0.00 & 0.00 & HR \\
\hline 90 & WGL1127 & 0.47 & 0.00 & $\mathrm{R}$ \\
\hline 91 & WGL1131 & 1.12 & 0.00 & $\mathrm{R}$ \\
\hline 92 & WGL1141 & 1.10 & 0.00 & $\mathrm{R}$ \\
\hline 93 & Tellahamsa & 21.08 & 12.42 & $\mathrm{~S}$ \\
\hline 94 & WGL32100 & 32.94 & 19.23 & HS \\
\hline 95 & RP1 & 7.14 & 0.76 & MS \\
\hline 100 & RP5587-B-B-B-32 & 23.87 & 15.38 & $\mathrm{~S}$ \\
\hline 101 & RP 2068-18-3-5 & 2.14 & 0.00 & MR \\
\hline 102 & JGL 20644 & 7.95 & 20 & $\mathrm{~S}$ \\
\hline 103 & JGL 21789 & 2.84 & 4.20 & MR \\
\hline 104 & JGL 21831 & 3.94 & 4.88 & MR \\
\hline 105 & JGL 24344 & 7.38 & 15.95 & $\mathrm{~S}$ \\
\hline 106 & JGL 24520 & 27.27 & 37.59 & HS \\
\hline 107 & JGL 25154 & 0.72 & 2.38 & MR \\
\hline 108 & JGL 25925 & 3.05 & 12.93 & $\mathrm{~S}$ \\
\hline 109 & JGL 25947 & 17.39 & 44.03 & HS \\
\hline 110 & JGL 25958 & 13.70 & 21.69 & $\mathrm{~S}$ \\
\hline 111 & JGL 25960 & 20.11 & 40.56 & HS \\
\hline 112 & JGL 25964 & 15.43 & 40.61 & $\mathrm{HS}$ \\
\hline 113 & JGL 25969 & 5.13 & 4.86 & MR \\
\hline 114 & JGL 25975 & 13.33 & 17.17 & $\mathrm{~S}$ \\
\hline 115 & JGL 25998 & 10.28 & 23.53 & $\mathrm{~S}$ \\
\hline 116 & JGL 26772 & 4.32 & 9.35 & MS \\
\hline 117 & JGL 26960 & 11.34 & 21.60 & $\mathrm{~S}$ \\
\hline 118 & JGL 26989 & 18.01 & 48.57 & HS \\
\hline 119 & JGL 27015 & 1.17 & 3.01 & MR \\
\hline 120 & JGL 27020 & 2.53 & 0.74 & MR \\
\hline 121 & JGL 27056 & 1.79 & 4.76 & MR \\
\hline 122 & JGL 27058 & 0.00 & 0.83 & $\mathrm{R}$ \\
\hline 123 & JGL 27063 & 1.76 & 7.63 & MS \\
\hline 124 & JGL 27072 & 13.04 & 35.76 & HS \\
\hline
\end{tabular}




\begin{tabular}{|c|c|c|c|c|}
\hline 125 & JGL 27075 & 4.47 & 3.76 & MR \\
\hline 126 & JGL 27143 & 5.38 & 24.38 & $\mathrm{~S}$ \\
\hline 127 & JGL 27353 & 6.56 & 6.29 & MS \\
\hline 128 & JGL 27356 & 2.72 & 5.92 & MS \\
\hline 129 & JGL 27361 & 2.72 & 2.91 & MR \\
\hline 130 & JGL 27371 & 0.00 & 1.63 & MR \\
\hline 131 & JGL 27391 & 11.59 & 25.00 & S \\
\hline 132 & KNM 1592 & 0.66 & 4.07 & MR \\
\hline 133 & KNM1598 & 1.23 & 4.14 & MR \\
\hline 134 & KNM 1600 & 2.50 & 6.21 & MS \\
\hline 135 & KNM 1610 & 4.60 & 10.06 & MS \\
\hline 136 & KNM 1638 & 4.62 & 8.90 & MS \\
\hline 137 & KNM 1616 & 3.29 & 8.61 & MS \\
\hline 138 & KNM 1621 & 3.92 & 4.39 & MR \\
\hline 139 & KNM 1625 & 1.72 & 6.47 & MS \\
\hline 140 & KNM 1632 & 1.23 & 8.39 & MS \\
\hline 141 & KNM 1717 & 19.12 & 44.44 & HS \\
\hline 142 & KNM 1722 & 1.32 & 5.66 & MS \\
\hline 143 & KNM1724 & 0.67 & 7.50 & MS \\
\hline 144 & KNM1728 & 7.14 & 25.55 & HS \\
\hline 145 & KNM 1730 & 10.81 & 17.69 & $\mathrm{~S}$ \\
\hline 146 & KNM 2213 & 11.92 & 24.14 & $\mathrm{~S}$ \\
\hline 147 & KNM 2251 & 3.21 & 5.04 & MR \\
\hline 148 & KNM 2275 & 2.16 & 0.00 & MR \\
\hline 149 & KNM 2266 & 2.65 & 4.03 & MR \\
\hline 150 & JGL 13595 & 0.00 & 1.64 & MR \\
\hline 151 & JGL 3828 & 0.68 & 4.92 & MR \\
\hline 152 & KNM 1623 & 2.31 & 4.93 & MR \\
\hline 153 & KNM 1638 & 4.35 & 2.61 & MR \\
\hline 154 & WGL-401 & 1.29 & 5.76 & MS \\
\hline 155 & WGL-505 & 1.84 & 3.27 & MR \\
\hline 156 & WGL-667 & 28.77 & 35.41 & HS \\
\hline 157 & WGL-705 & 27.39 & 50.63 & HS \\
\hline 158 & WGL-767 & 16.38 & 34.09 & HS \\
\hline 159 & WGL-810 & 15.32 & 16.23 & $\mathrm{~S}$ \\
\hline 160 & WGL-819 & 13.13 & 9.68 & MS \\
\hline 161 & WGL-825 & 14.39 & 8.11 & MS \\
\hline 162 & WGL-938 & 16.15 & 4.60 & $\mathrm{~S}$ \\
\hline 163 & WGL-1062* & 13.29 & 5.95 & $\mathrm{~S}$ \\
\hline 164 & JGL1118 & 14.02 & 2.63 & $\mathrm{~S}$ \\
\hline
\end{tabular}

R-Resistant, MS-Moderately susceptible, S-Susceptible, HS-Highly susceptible

After extensive testing of host-plant differentials it is found that biotype 1 cannot damage entries containing resistance genes derived from either Eswarakora or Siam 29, biotype 2 can damage the Eswarakora group but is unable to damage the Siam 29 group, whereas biotype 3 can damage the Siam
29 group but not the Eswarakora group [4]. Although geographic distributions of different gall midge biotypes is complex. After testing of different rice entries it is found that gall midge biotype 1 is present in Sambalpur. Previously it is also reported that populations of Hyderabad, 
Warangal, and Maruteru in Andhra Pradesh, Sambalpur in Odisha, and Raipur in Chhattisgarh qualified to be biotype 1, populations of Cuttack and Bhubaneswar in Odisha and at Mangalore in Karnataka, in Goa and at Sakoli in Maharashtra qualified to be biotype 2. GM populations at Ranchi in Bihar and Wangbal in Manipur had biotype 3 characteristics [4]. North coastal districts of Srikakulam and Vizianagaram in Andhra Pradesh and the Bhandara (Sakoli) region of Maharashtra qualified to be biotype 4 populations. Moncompu area of Kerala qualified to be biotype 5. In Manipur where biotype 3 had prevailed earlier another biotype emerged. The standard set of host-plant differentials confirmed the existence of yet another new biotype, designated as biotype 6 [7].

Many resistant and moderately resistant varieties are being cultivated by the farming community in Odisha to reduce gall midge damage considerably. These include Heera, Kalinga-II, Neela, Tara, Khandagiri, Udaya, Daya, Gouri, Pratap, Shakti, Phalguna, Meher, Birupa, Bhanja, Pratiksha and Samanta for medium lands and Samalei, Manika and Urbashi for lowlands [2]. Sumathi and Manickam, [10] tested different rice accession in field condition at Rice Research Station, Tirur, Tamilnadu during 2009 and found that the cultures viz., RP 4683-29-2-645, RP 4683-30-1648, RP 4686-49-1- 943, RP 4687-52-2-1197, RP 4688-532-1258, RP 4688-53-2-1259, JGL 17025, JGL 17183, JGL 17187, JGL 17189, KAVYA, JGL 17190, JGL 17196, JGL 17198, JGL 17211 and JGL 17221 were recorded nil gall midge damage and found to be resistant in field screening.

\section{CONCLUSION}

The genotypes W 1263, INRC 3021, Sudu Hondarawala, PTB 26, RP4686-48-1-937, RMSG-11, WGL 1147, WGL 1127, WGL 1121, WGL 1131, WGL 1141, JGL 27058 exhibited resistance against gall midge so, they can be developed as varieties or can be used in breeding programme as a source of gall midge resistance.

\section{ACKNOWLEDGEMENT}

The authors are highly thankful to ICAR-Indian Institute of Rice Research, Rajendranagar, Hyderabad and Orissa University of Agriculture and Technology, Bhubaneswar for financial assistance.

\section{REFERENCES}

[1] Bentur, J. S., Pasalu, I. C., Kalode, M. B. 1992. Inheritance of virulence in rice-gall midge (Orseoliaoryzae). Indian Journal of Agricultural Sciences, 62: 492-493.
[2] Dash, A. N. 2004. The rice gall midge problem in Orissa. In : New Approaches to Gall Midge Resistance in Rice, J. Bennett, J. S. Bentur, I. C. Pasalu and K. Krishnaiah (eds.). Proc. the International Workshop, 22-24November 1998, Hyderabad, India. LosBaños, Philippines. p. 195.

[3] Kalode, M. B., Viswanathan, P. R. 1976. Changes in relative pest status in insect pests in rice. Indian Journal of Plant Protection, 4: 79-91.

[4] Kalode, M. B. and Bentur, J. S. 1989. Characterization of Indian biotypes of the rice gall midge, Orseolia oryzae (Wood-Mason) (Diptera: Cecidomyiidae). International Journal of Tropical Insect Science, 10: 219-24.

[5] Krishnaiah, K. 2004. Rice gall midge, Orseolia oryzae-an overview. In : New Approaches to Gall Midge Resistance in Rice, J. Bennett, J. S. Bentur, I. C. Pasalu and K. Krishnaiah (eds.). Proc. International Workshop, 22-24 November 1998. Hyderabad, India. LosBaños, Philippines. p. 195.

[6] Pasalu, I. C., Katti, G. 2006. Advances in ecofriendly approaches in rice IPM. Journal of Rice Research, 1(1):83-90.

[7] Pasalu, I. C., Huang, B. C., Zang, Y and Yu-Juan Tan, Y. J. 2004. Current status of rice gall midgebiotypes in India and China. In : New Approaches to Gall Midge Resistance in Rice, J. Bennett, J. S. Bentur, I. C. Pasalu and K. Krishnaiah (eds.). Proc. the International Workshop, 22-24 November 1998, Hyderabad, India. LosBaños, Philippines. p. 195.

[8] Rajamani, S., Pasalu, I. C., Mathur, K. C and Sain, M. 2004. Biology and ecology of rice gall midge. In : New Approaches to Gall Midge Resistance in Rice, J. Bennett, J. S. Bentur, I. C. Pasalu and K. Krishnaiah (eds.). Proc.the International Workshop, 22-24 November 1998, Hyderabad, India. LosBaños, Philippines. p. 195.

[9] Seni, A., Naik, B. S. 2017. Efficacy of some insecticides against major insect pests of rice, Oryza sativa L. Journal of Entomology and Zoology Studies, 5 (4): 1381-1385.

[10] Sumathi, E., Manickam, G. 2013. Field screening of rice accessions against rice gall midge (Orseolia oryzae Wood-Mason). Crop Research, 45: 54-58.

[11] Ukwungwu, M. N., Williams, C. T. and Okhldlevble, O. 1999. Screening of African rice, Oryza glaberrima Steud for resistance to rice gall midge, Orseolia oryzivora Harris and Gagne. Journal of Food Technology in Africa, 4 : 108-10. 